\title{
ON THE SMALL DIVISORS IN THE LUNAR THEORY*
}

\author{
BY
}

\section{ERNEST WILLIAM BROWN}

\section{INTRODUCTION.}

1. One of the greatest difficulties which occur in the actual calculations necessary to obtain the coefficients of the periodic terms which express the coordinates of the Moon in terms of the time, is the presence of small divisors. At each stage of the approximations either one or two such divisors enter in each set of terms, $\dagger$ and the entrance of a small divisor means, in general, a loss of accuracy in the corresponding coefficient. To illustrate the manner in which these divisors enter, it is sufficient to refer to the general forms which the equations of motion take. For example, consider the equations

$$
\begin{aligned}
\ddot{x}+x & =\sum_{i} a_{i} \cos (2 i+a) t, \quad(i=0, \pm 1, \pm 2, \cdots), \\
\dot{y} & =\sum_{i} b_{i} \cos (2 i+a) t
\end{aligned}
$$

where $a_{i}, b_{i}, a$ are constants, $a$ being nearly unity or nearly zero.

The particular integrals are

$$
\begin{aligned}
& x=\sum \frac{a_{i}}{1-(2 i+a)^{2}} \cos (2 i+a) t, \\
& y=\sum \frac{b_{i}}{2 i+a} \sin (2 i+a) t .
\end{aligned}
$$

The small divisors occur for

$$
\begin{array}{ccccc}
i=0,-1 & \text { in } x \text { when } & a=1 & \text { nearly, } \\
i=0 & \text { in } y & \text { when } & a=0 & \text { nearly. }
\end{array}
$$

The corresponding coefficients are increased in size. If a numerical development is being made, the number of places of decimals to which the results are accurate will be less in the integrals than in the differential equations. If a literal development is under consideration such that $a_{i}, b_{i}, a$ are series in positive integral powers of a small quantity $m$, and if $a$ or $1-a$ be divisible by

* Presented to the Society February 22, 1902. Received for publication February 4, 1902.

$+A$ 'set of terms' is defined in Article 10 below. 
$m$, the particular coefficients referred to will be known to one less power of $m$ than the corresponding coefficients in the differential equations.

2. Now it is well known that, for all inequalities depending on the attractions of the sun and earth alone, ${ }^{*}$ whatever small divisors may occur, the results never contain negative powers of $m$, where $m$ is the ratio of the mean motions of the sun and moon. $\dagger$ Hence, if a small divisor contains the factor $m^{p}$, the corresponding coefficients $a_{i}, b_{i}$ must also contain this factor.

The coefficients of the periodic terms in each approximation depend on the results of previous approximations and there is, in general, a loss of accuracy at each stage, which arises in the following way. Suppose that a coefficient in the differential equations appears in the form

(a) $\left(b_{0}+b_{1} m+b_{2} m^{2}+\cdots+b_{p} m^{p}\right)-\left(b_{0}+b_{1} m+b_{2}^{\prime} m^{2}+\cdots+b_{p}^{\prime} m^{p}\right)$,

where the $b_{i}, b_{i}^{\prime}$ are rational numerical fractions, i. e., it appears as the differ. ence of two expressions which are known to $m^{p}$. If the divisor be $m$, the result is only known to $m^{p-1}$.

If, however, the calculations could have been so arranged that the coefficient appeared in the form

$$
\left(b_{1} m+b_{2} m^{2}+\cdots+b_{p} m^{p}\right)\left(b_{1}^{\prime} m+b_{2}^{\prime} m^{2}+\cdots+b_{p}^{\prime} m^{p}\right),
$$

the result after division by $m$, would still be true to $m^{p}, \ddagger$ that is, there would have been no loss of accuracy.

3. If the numerical value of $\mathrm{m}$ be used from the start, similar results hold; instead of considering powers of $\mathrm{m}$, we consider the number of places of decimals. The question of accuracy is a little more complicated since the coefficients of the higher powers of $\mathrm{m}$ are frequently large numbers, that is, the series arranged in powers of $m$ converge slowly in many cases. But the gain in accuracy is great since $m$ only occurs in the original differential equations as a factor to the first and second powers, so that we exchange a slowly converging (or perhaps diverging) series in powers of $\mathrm{m}$ for a series $\sum b_{i} 10^{-i}$ where $b_{i}$ is a positive integer less than 10.

In the lunar theory $m$ is about $\frac{1}{13}$ and the small divisors are generally infinite series of positive powers of $\mathrm{m}$, beginning with the first power. The loss of accuracy caused by such a divisor at each stage of the approximations is gener-

* The ratio of the mass of the moon to that of the sun is supposed to be neglected.

+ In this theory $m$ is the ratio $n^{\prime} /\left(n-n^{\prime}\right)$, where $n^{\prime}, n$ are the mean motions. The statement is true for either $m$ or $\mathrm{m}$.

$\ddagger$ The phrases "true to $m$ " or "accurate to $m^{\prime}$ " are taken throughout to mean that all coefficients up to and inclusive of that of $m^{p}$ are accurately known. A similar meaning is attached to the phrase "accurate to $p$ places of decimals." 
ally balanced by the presence of a new small factor independent of $\mathrm{m}$ and smaller than $\mathrm{m}$; this factor is one of the four quantities : $\mathrm{e}=$ the eccentricity of the moon's orbit, $e^{\prime}=$ that of the sun's orbit, $\mathrm{k}=$ the inclination of the moon's orbit, $a=$ the ratio of the mean distances of the moon and sun. The numerical accuracy would be the same at any stage of the approximations as it is at the start were it not for two circumstances. First, at every stage of the approximations after the second (that is, when the order with reference to e, $e^{\prime}, \mathrm{k}, a$ is greater than the first) there are one or more terms having as divisors series which begin with $\mathrm{m}^{2}$; second, the numerical multiplier of the lowest power of $\mathrm{m}$ present in a coefficient may be large, and these multipliers tend, in general, to increase with the order of the term. These facts require the earlier approximations to be taken to a higher degree of accuracy than would otherwise have been necessary. There are no divisors beginning with $\mathrm{m}^{3}$ within the practical limits set by observation.* The loss of accuracy due to a large numerical multiplier of the lowest power of $m$ is judged by the ratio of the numerical value of the coefficient to $\lambda \mathrm{m}^{q}$ where $\lambda$ is the characteristic $\dagger$ and $\mathrm{m}^{q}$ the lowest power of $\mathrm{m}$ present.

When the literal value of $m$ is used all the multipliers of the different powers of $m$ are rational numerical fractions. If the numerical value of $m$ be used from the outset, $m$ being a quantity obtained from observation to a certain number of places of decimals, there is a loss of accuracy due to the use of a finite number of places of decimals in long and complicated calculations. I have examined elsewhere $\ddagger$ the maximum loss which can be produced from this cause and have shown that when the number of operations (addition, multiplication, etc.) is less than 63 the loss is not more than two places of decimals. As a matter of fact, however, the actual loss will never be more than this, however extended be the calculations, since positive and negative errors tend to balance one another.

4. The main object of this paper is to show how the method $\S$ now being used for the calculation of the inequalities produced by the attraction of the sun can be modified so that the total loss of accuracy, as far as the small quantity $\mathrm{m}$ is concerned, does not exceed $q-2$ powers of $\mathrm{m}$ when the 'order of the characteristic is $q$, with the exception of certain coefficients of the second and third orders.

This theorem is proved for a literal expansion in positive powers of $\mathrm{m}, \mathrm{e}, e^{\prime}, \mathrm{k}, a$. From what has been said above it is not difficult to make an

* The largest coefficient with such a divisor has the factor $\mathrm{ek}^{2} e^{\prime 4} \mathrm{~m}$ at least. In Delaunay's notation this term has the argument $l+2 \mathrm{~F}-4 \mathrm{D}-4 l^{\prime}$, and the loss of accuracy for it is only one power of $\mathrm{m}$. Cf. Art. 14, etc.

$\dagger$ The characteristic is defined in Art. 10.

† Haverford College Studies, no. 11 (1892).

§ T. M. M., part I. See footnote 1 on page 164 . 
estimate of the degree of numerical accuracy required at the outset for a given degree of numerical accuracy in all coefficients.

First, e, $e^{\prime}, \mathrm{k}, a$ are all less than $\frac{1}{10}$, so that a characteristic of order $q$ contains a factor $10^{-q}$ at least. Second, large numerical multipliers cause a loss of two places of decimals at most within the limits set by observation. Third, an estimated loss of two places will occur owing to the use of decimals. Hence, within the limits set by observation, this maximum loss of accuracy in the terms of order $q$ is

$$
q-2-q+2+2=2
$$

The two quantities which require to be most accurately known for comparison with observation are the mean motions of the perigee and node and it is useful to have the ratio of these to the mean motion of the moon accurately to 9 places of decimals. It is not necessary to know the numerical coefficients of the periodic terms to more than 8 places. The intermediate orbit-HiLL's variation curve*-used in the theory is the first approximation. In finding the principal parts of the motions of the node and perigee there is a gain of one power of $m, \dagger$ and an estimated loss of two places of decimals owing to extended calculations over the results in the variation orbit-a net loss of one place. Hence, for these mean motions we require the variation orbit (which contains no small divisors) to $9+1$ places, and, from the statement above, for the the coefficients of the periodic terms it should be known to $8+2$ places. Hence, for all purposes it is sufficient to know the coefficients in the intermediate orbit to 10 places of decimals.

5. In order to clear the way for the proof of the theorem enunciated in Art. 4, a general formula is given in Sect. I for the gain or loss of accuracy in such expressions as actually occur in the theory. In Sect. II will be found the differential equations in various forms and an investigation of the expressions which are affected by small divisors. Sect. III contains certain transformations which assist in preventing a loss of accuracy. In Sect. IV the detailed discussion necessary to prove the theorem for the first four orders is given with a general indication of the proof for higher orders.

It is quite possible that transformations might be found such that no loss of accuracy, as far as powers of $\mathrm{m}$ are concerned, occurs. Such transformations would doubtless be somewhat complicated and difficult to arrange for computation. Moreover, from the result at the end of Art. 4, they are not practically necessary and it does not seem useful to extend analytical transformations, already somewhat long, beyond a point where theoretical or practical interest ceases.

* G. W. HiLL, Researches in the Lunar Theory: A merican Journal of Mathematics, vol. 1 (1878).

†G. W. HrLc, Literal expression for the motion of the IIoon's Perigee: A nnals of Mathematics, vol. 9 (1891). The same result is obviously true for the motion of the node. 


\section{Section I. A criterion of aCcuract.}

6. Consider the expression

$$
\sum \frac{S_{1} S_{2} \cdots S_{p}}{T_{1} T_{2} \cdots T_{q}^{\prime}} \equiv \sum \frac{\Pi S_{i}}{\Pi T_{i}}
$$

where $S_{i}, T_{i}$ are power series in $\mathrm{m}$, each of them known up to some definite power which may differ in the different series, the coefficients in the series being rational numerical fractions independent of $m$, and where $\sum$ denotes a sum of such expressions. It is desired to find the highest power of $m$ whose coefficient is accurate when the expression is expanded in powers of $\mathrm{m}$.

As there is no need to consider the numerical coefficients in the power series, here and in the following sections $(a, \beta)$ will be used to denote a power series in which the first power of $m$ whose coefficient is not zero is $m^{\alpha}$ and in which the greatest known power of $m$ is $\mathrm{m}^{\beta}$. In accordance with this notation, put

$$
\begin{aligned}
S_{i} & =\left(a_{i}, \beta_{i}\right), \quad T_{i}=\left(\gamma_{i}, \delta_{i}\right), \\
\Pi S_{i} \div \Pi T_{i} & =\Pi\left(a_{i}, \beta_{i}\right) \div \Pi\left(\gamma_{i}, \delta_{i}\right)=(A, B) .
\end{aligned}
$$

It is desired to find $B$. When $B$ is known for each such expression, the required number is the least of the numbers $B$ present in $\sum$. The second number in any parenthesis will be called the degree of uccuracy.

Suppose that any one of the series is known to any power of $\mathrm{m}$, i. e., that the coefficients proceed according to a known law, or that its accuracy is greater than any we need to consider; we can signalize the fact by putting $\infty$ for the second number in the bracket. Then

$$
(a, \infty)=\mathrm{m}^{a}(0, \infty),
$$

and as $(0, \infty)$ does not affect the accuracy of the result, we can collect all such terms in the numerator and denominator and consider the expression

$$
\mathrm{m}^{k} \frac{\left(a_{1}, \beta_{1}\right) \cdots\left(a_{p}, \beta_{p}\right)}{\left(\gamma_{1}, \delta_{1}\right) \cdots\left(\gamma_{q}, \delta_{q}\right)}=(A, B) .
$$

First, noting the lowest power of $m$ occurring in each series, we have

$$
A=\sum a-\sum \gamma+k .
$$

Second, the number of terms in $(A, B)$ cannot be greater than the number of terms in any one of the component series. Hence

$$
B-A=\text { the least of } \beta_{i}-a_{i}, \delta_{i}-\gamma_{i} .
$$

These results determine $A, B$. 
7. In the applications to be made the following limitations may be imposed :

(i) For all factors of the denominator in which $\gamma_{i}=0, \delta_{i}-\gamma_{i}$ is equal to or greater than any of the other $\delta_{i}-\gamma_{i}$ or than any of the $\beta_{i}-a_{i}$;

(ii) The factors of the denominator in which $\gamma_{i} \neq 0$, are at most two in number and then of the form $(\gamma, \delta)^{2}$;

(iii) $a_{i}, \gamma$ are positive;

(iv) $\gamma<3$.

Thus the expression reduces to

and in this

$$
\mathrm{m}^{k}\left(a_{1}, \beta_{1}\right) \cdots\left(a_{p}, \beta_{p}\right) \div(\gamma, \delta)^{q}=(A, B) \quad(q=0,1,2),
$$

$$
A=k-q \gamma+\sum a,
$$

$$
B-A=\text { the least of the numbers } \beta_{i}-a_{i}, \delta-\gamma \text {. }
$$

(v) The numbers $a_{i}, \beta_{i}, A, B$ will denote the numbers set down or any respectively greater numbers, but $\gamma, \delta$ always denote the definite numbers set down.

Section III. The differential equations and their solution By apProximation. SMall Divisors.

8. The differential equations. The notation adopted in the series of memoirs on the motion of the moon* will be in general used here. The equations of motions are $\dagger$

$$
\left\{\begin{aligned}
\text { a. } & D^{2} u+2 \mathrm{~m} D u & =\frac{\partial f}{\partial s}, \\
\text { b. } & D^{2} s-2 \mathrm{~m} D s & =\frac{\partial f}{\partial u}, \\
\text { c. } & D^{2} z & =\frac{1}{2} \frac{\partial f}{\partial z},
\end{aligned}\right.
$$

where $x, y, z$ are the coördinates of the moon's center and

$$
\begin{gathered}
u=x+y \sqrt{-1}, \quad s=x-y \sqrt{-1}, \quad r^{2}=x^{2}+y^{2}+z^{2}=u s+z^{2}, \\
D=-\frac{\sqrt{-1}}{n-n^{\prime}} \frac{d}{d t}, \quad \mathrm{~m}=\frac{n^{\prime}}{n-n^{\prime}},
\end{gathered}
$$

$n^{\prime}, n$ being the observed mean motions of the sun and moon. Also

* Ernist W. Brown, Theory of the Motion of the Moon, Memoirs of the Royal Astronomical Society, part I (1897) in vol. 53, pp. 39-116; part II (1899), pp. 163-202 ; part III (1900) in vol. 54, pp. 1-63. These will be referred to under the abbreviation "T. M. M., pts. I, II, III."

†T. M. M., part I, section (ii). 


$$
\left\{\begin{array}{l}
f=f_{-1}+f_{2}+f_{3}+\cdots=\sum_{p} f_{p} \quad(p=-1,2,3,4, \cdots), \\
f_{-1}=2 \kappa / r, f_{2}=f_{2}^{\prime}+f_{2}^{\prime \prime}, \\
\left.f_{2}^{\prime}=-\frac{3}{4} \mathrm{~m}^{2}(u+s)^{2}+\mathrm{m}^{2} z^{2}, \quad f_{2}^{\prime \prime}=e^{\prime} \mathrm{m}^{2} \text { (function of } u, s, t\right),
\end{array}\right.
$$

in which $f_{p}$ is a homogeneous function of $u, s, z$ of degree $p$ containing only even powers of $z$, and always multiplied by the factor $\mathrm{m}^{2}$ except when $p=-1$. Denoting by $a_{0}, a^{\prime}$ the mean distances of the moon and sun used in the theory,

$$
\kappa=(1+2 \mathrm{~m}+\cdots) a_{0}^{3},
$$

and $f_{p}$ contains the factor $\left(a_{0} / a^{\prime}\right)^{p-2}=a^{p-2}$ when $p>2 ; f_{p}$ also contains $t$ explicitly when $p \geqq 2$.

9. From the equations (1) the following are easily derived :*

(2)

$$
\left\{\begin{array}{l}
\text { a. } u D s-s D u-2 \mathrm{~m} u s=D^{-1}\left(u \frac{\partial f}{\partial u}-s \frac{\partial f}{\partial s}\right) \\
\text { B. } D u \cdot D s+(D z)^{2}=C+f-D^{-1}\left(D^{\prime} f\right) \\
C . D^{2}\left(u s+z^{2}\right)-2 D u \cdot D s-2(D z)^{2}-2 \mathrm{~m}(u D s-s D u)=\sum p f_{p}, \\
\text { d. } D^{2}\left(u s+z^{2}\right)-D u \cdot D s-(D z)^{2}-2 \mathrm{~m}(u D s-s D u) \\
=C+\sum(p+1) f_{p}-D^{-1}\left(D^{\prime} f\right), \\
\text { e. } \quad D(u D z-z D u)-2 \mathrm{~m} z D u=\frac{1}{2} u \frac{\partial f}{\partial z}-z \frac{\partial f}{\partial s}, \\
f . \quad D^{2}\left(u s+z^{2}\right)-D u \cdot D s-\left(D_{z}\right)^{2}+u D s-s D u-2 \mathrm{~m}(2 \mathrm{~m}+1) u s \\
\quad=C+\sum(p+1) f_{p}+D^{-1}\left\{(2 \mathrm{~m}+1)\left(u \frac{\partial f}{\partial u}-s \frac{\partial f}{\partial s}\right)-D^{\prime} f\right\}
\end{array}\right.
$$

g. $D^{2}\left(u s+z^{2}\right)-D u \cdot D s-(D z)^{2}+\frac{1}{2}(u D s-s D u)(1-\mathrm{m})-\mathrm{m}(1+3 \mathrm{~m}) u s$

$$
=C+\sum(p+1) f_{p}+D^{-1}\left\{\frac{1}{2}(1+3 \mathrm{~m})\left(u \frac{\partial f}{\partial u}-s \frac{\partial f}{\partial s}\right)-D^{\prime} f\right\},
$$

where $D^{\prime} f$ denotes the derivative of $f$ with respect to $\sqrt{-1}\left(n-n^{\prime}\right) t$ only in so far as $t$ is contained explicitly in $f, D^{-1}$ denotes the operation inverse to $D$, i. e., an integration with respect to $\sqrt{-1}\left(n-n^{\prime}\right) t$, and $C$ is a constant of integration. The equations $(2 B),(2 C)$, distinguished by capital letters alone contain $f_{-1}$. If the parallax of the sun be neglected $f=f_{-1}+f_{2}$ and the equa-

*T. M. M., pt. I, pp. 53, 54, contain equations $(2 a)-(2 e) ;(2 f),(2 y)$ are obvious consequences of these. 
tions with small letters are all of the second degree with respect to $u, s, z \mathrm{ex}$ cept for the constant $C$; if the eccentricity of the sun be neglected, $D^{\prime} f=0$; if both these constants be neglected $f=f_{-1}+f_{2}^{\prime}$ and the equations give all terms depending only on $\mathrm{m}$ and the eccentricity and inclination of the moon's orbit. Of these seven equations three only are necessary to replace equations (2) and one of the three must be (2e).

10. The values of $u, s, z$ derived from these equations are known to be formally expansible in cosines and sines of multiples of the angles $\left(n-n^{\prime}\right) t+t_{0}$, $n^{\prime} t+t_{1}, \bullet\left(n-n^{\prime}\right) t+t_{2}, \mathrm{~g}\left(n-n^{\prime}\right) t+t_{3}$, with coefficients expanded in ascending powers of $\mathrm{m}, \mathrm{e}, e^{\prime}, \mathrm{k}, a$, where $\mathrm{c}, \mathrm{g}$ are constants to be determined. If we put

$$
\zeta=e^{t\left(n-n^{\prime}\right) \sqrt{-1}},
$$

the periodic terms can be expressed in positive and negative powers of $b_{0} \zeta, b_{1} \zeta^{\mathrm{m}}$, $b_{2} \zeta^{\mathrm{o}}, b_{3} \zeta^{\mathrm{g}}$, where $b_{i}=e^{t_{i} v-1}=$ const. Since we are only concerned here with the determination of the coefficients of the periodic terms and since $\mathrm{m}, \mathrm{c}, \mathrm{g}$ are supposed to be incommensurable with unity and finally, since the method used is that of indeterminate coefficients, the constants $b_{i}$ may be omitted throughout and then all periodic terms are expressible in positive and negative powers of $\zeta, \zeta^{\mathrm{m}}, \zeta^{\mathrm{o}}, \zeta^{\mathrm{g}}$.

The general expressions for $u \zeta^{-1}, z \sqrt{-1}$ are then sums of terms of the form

$$
a_{0} \mathrm{e}^{i_{1}} e^{i_{2}} \mathbf{k}^{i_{3}} a^{i_{4}}\left(\lambda_{i} \zeta^{\tau+2 i}+\lambda_{-i}^{\prime} \zeta^{-\tau-2 i}\right)
$$

where

$$
\begin{aligned}
& \tau=\left(2 i_{1}^{\prime}-i_{1}\right) \mathrm{c}+\left(2 i_{2}^{\prime}-i_{2}\right) \mathrm{m}+\left(2 i_{3}^{\prime}-i_{3}\right) \mathrm{g} ; \\
& i_{j}, i_{j}^{\prime}=0,1,2, \ldots ; i_{j}^{\prime} \leqq i_{j} ; \\
& 2 i=0, \pm 1, \pm 2, \ldots ; 2 i-i_{4}=\text { even no.; }
\end{aligned}
$$

in $u \zeta^{-1}$, the values of $i_{3}$ are even,

$$
\text { in } z \sqrt{-1} \text {, the values of } i_{3} \text { are odd and } \lambda_{-i}^{\prime}=-\lambda_{i} \text {; }
$$

$a_{0}$ is the linear unit; $\lambda_{i}, \lambda_{-i}^{\prime}$ are positive power series in $\mathrm{m}$ for which the notation *

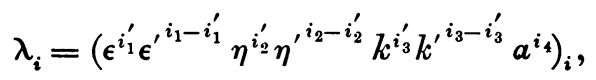

with $\lambda_{i}^{\prime}$ obtained by interchanging $\epsilon$ with $\epsilon^{\prime}, \eta$ with $\eta^{\prime}, k$ with $k^{\prime}$, is used except when $i_{j}, i_{j}^{\prime}$ are all zero when $\lambda_{i}=a_{i}$.

A set of terms contains all the terms for which $i_{j}, i_{j}^{\prime}$ are the same, i. e., the sum when $i$ only receives all values. The characteristic of the set is

* T. M. M., pt. I, p. 60. The form in which the notation is presented here has been slightly changed but its essential character is unaltered. The coefficients for terms of the first order are $(\varepsilon)_{i},\left(\varepsilon^{\prime}\right)_{i}, \cdots,(a)_{i}$; these are written $\varepsilon_{i}, \varepsilon_{i}^{\prime}, \cdots, a_{i}$, for the sake of brevity. 
$\mathrm{e}^{i_{1}} e^{i_{2}} \mathbf{k}^{i_{3}} a^{i_{4}}$, and the order of the characteristic or simply the order of the set is $i_{1}+i_{2}+i_{3}+i_{4}$. The argument of any term is the index of $\zeta$ in that term.

The calculations show that

$$
\mathbf{c}=1+\mathrm{m}-\frac{3}{4} \mathrm{~m}^{2}-\cdots, \quad g=1+\mathrm{m}+\frac{3}{4} \mathrm{~m}^{2}+\cdots,
$$

where the remaining parts consist of higher powers of $m$ and terms involving powers of $\mathrm{m}$ and powers and products of $\mathrm{e}^{2}, e^{\prime 2}, \mathrm{k}^{2}, a^{2}$. For our purpose, the first two terms in $\mathrm{c}, \mathrm{g}$ are alone of importance; the others all contain the factor $\mathrm{m}^{2}$ (see Art. 3).

11. The method of approximation consists in successively determining the terms of orders $0,1,2, \ldots$. A set of terms of any given order can be found independently of all other sets of the same or higher order, but the coefficients in any one set depend on one another in the forms which the equations take here, ${ }^{*}$ so that it is necessary to consider all the terms of a set together. Let

$$
u=u_{0}+u_{1}+u_{2}+\cdots, \quad z=z_{1}+z_{2}+\cdots,
$$

where $u_{\lambda}, z_{\lambda}$ contain all terms of order $\lambda$, and suppose that we wish to determine terms of order $\lambda(\lambda \neq 0)$ in $u$ or $z$, those of lower orders having been obtained.

Let $Q(u, s, z)$ be any one of the functions which we shall use, and let $[Q]_{\lambda}$ denote the terms of order $\lambda$ in $Q$ after expansion has taken place. Then

Put

$$
[Q]_{\lambda}=\left[Q\left(\sum_{\mu=0}^{\lambda} u_{\mu}, \quad \sum_{\mu=0}^{\lambda} s_{\mu}, \quad \sum_{\mu=1}^{\lambda} z_{\mu}\right)\right]_{\lambda} .
$$

$$
[Q]_{\lambda}^{\prime}=\left[Q\left(\sum_{\mu=0}^{\lambda-1} u_{\mu}, \quad \sum_{\mu=0}^{\lambda-1} s_{\mu}, \quad \sum_{\mu=1}^{\lambda-1} z_{\mu}\right)\right]_{\lambda},
$$

so that, after expansion by Taylor's theorem,

$$
[Q]_{\lambda}=[Q]_{0}+u_{\lambda}\left[\frac{\partial Q}{\partial u}\right]_{0}+s_{\lambda}\left[\frac{\partial Q}{\partial s}\right]_{0}+z_{\lambda}\left[\frac{\partial Q}{\partial z}\right]_{0}+[Q]_{\lambda}^{\prime} .
$$

The known terms are thus separated out from the unknown terms and all the equations will be linear with respect to the unknowns. All our functions are such that $Q$ contains either only even or only odd powers of $z$; in the former case the fourth term, in the latter case the first three terms are absent from $[Q]_{\lambda}$.

When this notation is applied to the equations (2) the remarks of Art. 8 show that $\quad\left[D^{-1}\left(D^{\prime} f\right)\right]_{\lambda}=\left[D^{-1}\left(D^{\prime} f\right)\right]_{\lambda}^{\prime}, \quad\left[f_{p}\right]_{\lambda}=\left[f_{p}\right]_{\lambda}^{\prime} \quad$ for $\quad p>2$.

* In a paper On the solution of a pair of simultaneous differential equations, etc. (M emoirs of the Cambridge Philosophical Society, vol. 18 (Stokes Memorial, 1900), pp. 94-106), I have shown how any coefficients in the set may be determined independently of all other coefficients of the same set when the terms of lower orders have been found. 
The symbol $P_{q}$ will be used as an abbreviation of the phrase "a positive ascending power series in $\mathrm{m}$ beginning with the $q$ th power of $\mathrm{m} . "$ The letter $P$ (without a suffix) stands for a similar abbreviation with the addition " $q \geqq 1$."

12. The first approximation consists of the terms of zero order and is given by

$$
u_{0} \zeta^{-1}=\sum_{i} a_{i} \zeta^{2 i}
$$

$$
(i=0, \pm 1, \pm 2, \cdots)
$$

in which $a_{0}$ is a certain length which we shall here put equal to unity, and $a_{i}=P_{|2 i|} \cdot$ Also

$$
f_{0}=-2-4 \mathrm{~m}+P_{2}, \quad u_{0} \frac{\partial f_{0}}{\partial u_{0}}=1+2 \mathrm{~m}+P_{2}, \quad s_{0} \frac{\partial f_{0}}{\partial s_{0}}=1+2 \mathrm{~m}+P_{2} .
$$

The set of terms with a characteristic of order $\lambda$ and arguments $\pm(\tau+2 i)$ are, for $u$, given by

$$
u \zeta^{-1}=\sum_{i}\left(\lambda_{i} \zeta^{\tau+2 i}+\lambda_{i}^{\prime} \zeta^{-\tau-2 i}\right) \quad(i=0, \pm 1, \pm 2, \cdots),
$$

where the characteristic is omitted. Substitute

$$
u=\sum_{\mu=0}^{\lambda} u_{\mu}, \quad z=\sum_{\mu=1}^{\lambda-1} z_{\mu}
$$

in equations (2) except $(2 e)$ and equate to zero the coefficient* of $\zeta^{\tau+2 i}$. The various terms in the coefficient may be divided into three classes. The first class consists of the terms containing $a_{0} \lambda_{i}, a_{0} \lambda_{-i}^{\prime}$, or $\lambda_{i}, \lambda_{-i}^{\prime}$ since $a_{0}=1$; the second class consists of terms of the form $\lambda_{j} P_{q}, \lambda_{j}^{\prime} P_{q}(q \geqq 2, j=0, \pm 1, \pm 2, \cdots)$, since $a_{i}=P_{|2 i|}$; the third class consists of known terms which do not involve $\lambda_{j}, \lambda_{j}^{\prime}$. The terms in the first class are those of principal importance in the determination of $\lambda_{i}, \lambda_{-i}^{\prime}$, since on them depends the existence or non-existence of small divisors.

For brevity write $\dagger$

$$
\lambda, \lambda^{\prime}, \tau \quad \text { instead of } \quad \lambda_{i}, \lambda_{-i}^{\prime}, \tau+2 i ;
$$

then the terms of principal importance in equations (2) are respectively

$$
\begin{cases}a . & -\tau\left(\lambda-\lambda^{\prime}\right)-2(1+\mathrm{m})\left(\lambda+\lambda^{\prime}\right), \\ B . & -\tau\left(\lambda-\lambda^{\prime}\right)-2(1+\mathrm{m})\left(\lambda+\lambda^{\prime}\right), \\ C . & 2 \tau(1+\mathrm{m})\left(\lambda-\lambda^{\prime}\right)+\left(\tau^{2}+3+6 \mathrm{~m}\right)\left(\lambda+\lambda^{\prime}\right), \\ d . & \tau(1+2 \mathrm{~m})\left(\lambda-\lambda^{\prime}\right)+\left(\tau^{2}+1+4 \mathrm{~m}\right)\left(\lambda+\lambda^{\prime}\right), \\ f . & 0\left(\lambda-\lambda^{\prime}\right)+\left(\tau^{2}-1-2 \mathrm{~m}\right)\left(\lambda+\lambda^{\prime}\right), \\ g . & \frac{1}{2}(1+\mathrm{m}) \tau\left(\lambda-\lambda^{\prime}\right)+\tau^{2}\left(\lambda+\lambda^{\prime}\right) .\end{cases}
$$

* The coefficients of $\zeta^{ \pm(\tau+2 i)}$ are equal in the equations.

$\dagger$ The fact that the symbol $\lambda$ has heen previously used for the characteristic does not canse any confusion, and these sbbreviations are of considerable advantage. 
These results show that if any pair of the equations (2), excluding (2e) and the pair $(2 a),(2 B)$, be used, the determinant of a pair of equations for finding $\lambda, \lambda^{\prime}$ is, except as to a constant factor which is not small,

$$
\tau\left(\tau^{2}-1-2 \mathrm{~m}\right),
$$

and this is equal to $P_{q}$ if either

$$
\tau=P_{q}, \quad \pm \tau=1+\mathrm{m}+P_{q} \quad(q=1 \text { or } 2) .
$$

I have shown that the more accurate statement* of the case is that small divisors equal to $P_{q}$ occur if, and only if,

$$
\tau=P_{q}, \quad \pm \tau=\mathrm{c}+P_{q},
$$

but the above statement is sufficient since $\mathrm{c}=1+\mathrm{m}+P_{2}$ and since we do not consider values of $q$ greater than 2 .

13. Three theorems will now be proved, two of which are necessary for the discussion which follows.

Theorem I. If $\pm \tau=1+\mathrm{m}+P_{q}$, then $3 \lambda+\lambda^{\prime}$ can be determined without loss of accuracy due to the small divisor.

For by $(3 a),(3 d)$, the equations $(2 a),(2 d)$ determine

$$
\begin{gathered}
-(1+\mathrm{m})\left(3 \lambda+\lambda^{\prime}\right)-\left(\lambda-\lambda^{\prime}\right)(\tau-1-\mathrm{m}), \\
(1+2 \mathrm{~m})\left(3 \lambda+\lambda^{\prime}\right)+(1+2 \mathrm{~m})\left(\lambda-\lambda^{\prime}\right)(\tau-1-\mathrm{m})+P_{2},
\end{gathered}
$$

respectively. Hence multiplying $(2 a)$ by $1+2 \mathrm{~m}$ and subtracting from $(2 d)$, we shall have an equation to determine $(1-\mathrm{m})\left(3 \lambda+\lambda^{\prime}\right)$. All the other terms containing $\lambda, \lambda^{\prime}$ in these equations have the factor $P_{2}$ at least, and therefore, since $q \leqq 2$, there is no loss of accuracy in the determination of $P_{2} \lambda, P_{2} \lambda^{\prime}$ and therefore none in that of $3 \lambda+\lambda^{\prime}$.

Theorem II. If $\tau=P_{q}$, then $\lambda+\lambda^{\prime}$ can be determined without loss of accuracy due to the small divisor.

For $(3 f),(2 f)$ show that the first approximation to $\lambda+\lambda^{\prime}$ has this property and $(3 B),(2 B)$ show that then $\lambda-\lambda^{\prime}$ has the divisor $\tau$. But the other terms in (2f) containing $\lambda, \lambda^{\prime}$ have the factor $P_{2}$ at least, and there is no loss of accuracy in $P_{2} \lambda$ or $P_{2} \lambda^{\prime}$.

* Investigations in the Lunar Theory, A merican J o u rnal of Mathematics, vol. 17 (1895), p. 356. This statement involves the divisor $\tau^{2}=P_{2 q}$ when $\tau=P_{q}$. Moreover, the right hand member of equation (2a) involves the divisor $\tau$. But whenever $\lambda, \lambda^{\prime}$ are contained in the right hand member of $(2 a)$ they occur with the factor $\mathrm{m}^{2} a_{ \pm 1} \div \tau=P_{4-q}$ which is equal to $P_{2}$ in the worst case $(q=2)$ and we are neglecting $P_{2}$ in the terms of principal importance. It might still be thought that when $q=2$, the terms involving $\mathrm{m}^{2}$ might disappear in the determinant, so as to make the divisor equal to $P_{3}$. That this is not the case I shall show in a future paper. 
Theorem III. All the coefficients in $1 / r$ for which $\tau=P_{1}$ are equal to $(2, \beta)$ and those for which $\tau=P_{2}$ are equal to $(1, \beta)$.

If $\tau=P_{1},(2 a)$ shows that $u D s-s D u=(1, \beta) ;(2 d)$ then shows that $D u \cdot D s+(D z)^{2}=(2, \beta) ;(2 B)$ then shows that $f=(2, \beta)$. The result follows since

$$
f=f_{-1}+(2, \beta)=(1+2 \mathrm{~m}) / r+(2, \beta) .
$$

If $\tau=P_{2},(2 d)$ shows that $D u \cdot D s+(D z)^{2}=(1, \beta)$ and thence, as before, from $(2 B), 1 / r=(1, \beta)$.

These three theorems show that the functions which may be found without loss of accuracy are

$$
\begin{aligned}
& 3 \lambda+\lambda^{\prime}, \quad\left(\tau^{2}-\mathrm{c}^{2}\right)\left(\lambda+\lambda^{\prime}\right) \quad \text { for } \pm \tau=\mathrm{c}+P_{q} ; \\
& \lambda+\lambda^{\prime}, \quad \tau\left(\lambda-\lambda^{\prime}\right), \quad 1 / r=P_{3-q} \text { for } \tau=P_{q} \\
& (q=1 \text { or } 2) \text {. }
\end{aligned}
$$

The degree of accuracy here referred to is that to which the known terms in the equation have been previously found.

14. The special portions of the equations whose accuracy is affected by the small divisors. There are four cases to consider

$$
\tau=P_{q}, \quad \tau=1+\mathrm{m}+P_{q} \quad(q=1 \text { or } 2) .
$$

The case $-\tau=1+\mathrm{m}+P_{q}$ is the same as $\tau=1+\mathrm{m}+P_{q}$ since the change $-\tau$ for $\tau$ merely alters the sign of $\lambda-\lambda^{\prime}$. In all cases $C$ is absent.

(i) $\tau=P_{1}$. Equation $(2 g)$ determines $\tau\left(\lambda-\lambda^{\prime}\right)$ without loss of accuracy. Also the right hand member contains the factor $\mathrm{m}^{2} \div \tau=P_{1}$ at the worst and is therefore divisible by $\tau$ without loss of accuracy. The only terms in the right hand member which are not divisible by $\tau$ without loss of accuracy are

$$
\begin{gathered}
{\left[-D u \cdot D s-(D z)^{2}+\frac{1}{2}(u D s-s D u)\right]_{\lambda}} \\
=\left[-D\left(u \zeta^{-1}\right) \cdot D(s \zeta)-\frac{1}{2} u \zeta^{-1} D(s \zeta)+\frac{1}{2} s \zeta D\left(u \zeta^{-1}\right)-(D z)^{2}\right]_{\lambda}
\end{gathered}
$$

for $D^{2}\left(u s+z^{2}\right)$ gives the factor $\tau^{2}$ and the terms other than those just written have the factor $m$.

The problem here is so to express (4) or $(4)^{\prime}$ that $\mathrm{m}$ or $P_{1}$ appears as a factor explicitly ; on division by $\tau$, the degree of accuracy then remains the same provided this factor itself is sufficiently accurate.

(ii) $\tau=P_{2^{*}}$. It would here be necessary to transform all terms in $(2 g)$ except $I^{2}\left(u s+z^{2}\right), 3 \mathrm{~m}^{2} u s, \sum(p+1) f_{p}$ which contain factors $\tau^{2}, \mathrm{~m}^{2}, \mathrm{~m}^{2}$ respectively, for $D^{-1}$ produces a divisor $P_{2}$, and the final division by $\tau$ produces another such divisor. A special transformation is given in Art. 17 for this case; it will also serve for the previous case. 
(iii) $\tau-\mathrm{c}=P_{1}$ or $P_{2}$. Equation $(2 f)$ determines

$$
\left(\tau^{2}-1-2 \mathrm{~m}\right)\left(\lambda+\lambda^{\prime}\right)=P_{q}\left(\lambda+\lambda^{\prime}\right)
$$

without loss of accuracy. The right hand member has the factor $\mathrm{m}^{2}$ and is therefore divisible by $\boldsymbol{P}_{2}$ without loss of accuracy. The portion of the left hand member which does not contain $\mathrm{m}^{2}$ explicitly is

$$
\left[D^{2}\left(u s+z^{2}\right)-D u \cdot D s-(D z)^{2}+u D s-s D u-2 \mathrm{~m} u s\right]_{\lambda},
$$

or, since $D^{2}$ produces $\tau^{2}$ and $\tau^{2}=1+2 \mathrm{~m}+P_{q}$,

$$
\begin{gathered}
{\left[-D u \cdot D s+u D s-s D u+u s-(D z)^{2}+D^{2}\left(z^{2}\right)\right]_{\lambda}} \\
=-\left[D\left(u \zeta^{-1}\right) \cdot D(s \zeta)+(D z)^{2}-D^{2}\left(z^{2}\right)\right]_{\lambda},
\end{gathered}
$$

the terms which are explicitly divisible by $\tau-\mathrm{c}=P_{q}$ being omitted. This last is the expression to be transformed so as to show the factor $P_{q}$ explicitly.

15. The previous results only refer to the terms in $u$; we have still to consider the equation for $z$. Consider equations $(1 c),(2 e)$ and choose out the terms of order $\lambda$. The terms of principal importance for the determination of the coefficient of $\zeta^{\tau}$ are obtained by substituting

$$
z_{\lambda} \sqrt{-1}=\lambda \cdot\left(\zeta^{\tau}-\zeta^{-\tau}\right), \quad u=u_{0},
$$

and equating to zero the coefficient of $\zeta^{ \pm \tau}$ in $(1 c)$ or of $\zeta^{ \pm \tau+1}$ in $(3 e)$. Powers of $m$ beyond the first being neglected, the equations give, for the terms of principal importance,

$$
\left(\tau^{2}-1-2 \mathrm{~m}\right) \lambda \text {. }
$$

There is thus a divisor $P_{q}$ when $\tau=1+\mathrm{m} \pm P_{q}$. The same remark as that made in Art. 12 applies here also, but with this difference: that the small divisor approximates to $\tau^{2}-\mathrm{g}^{2}$ instead of $\tau^{2}-\mathrm{c}^{2}$. This difference, however, does not affect our argument, since $\mathrm{c}, \mathrm{g}$ only differ by $\boldsymbol{P}_{2}$ and we are not considering divisors equal to $P_{3}$.

\section{I shall now prove the}

TheOREM.-All coefficients in z can be determined without loss of accuracy due to small divisors in the equation for $z$.

The only cases where there may be a loss of accuracy are in those coefficients where $\pm \tau=1+\mathrm{m}+P_{q}(q=1$ or 2$)$.

Consider equation $(3 e)$. The only terms which do not contain $\mathrm{m}^{2}$ explicitly are

$$
[\cdot D(u D z-z D u)-2 \mathrm{~m} z D u]_{\lambda} .
$$

$1^{\circ}$. Suppose $\tau=1+\mathrm{m}+P_{q}$. Equate to zero the coefficient of $\zeta^{1-\tau}$. Then $(a)$ becomes, for this coefficient, 


$$
\begin{aligned}
& {\left[-\left(\mathrm{m}+P_{q}\right)(u D z-z D u)-2 \mathrm{~m} z D u\right]_{\lambda}, } \\
= & {\left[-P_{q}(u D z-z D u)-\mathbf{m} D(u z)\right]_{\lambda} } \\
= & {\left[-P_{q}(u D z-z D u)+\mathbf{m}\left(\mathrm{m}+P_{q}\right) u z\right]_{\lambda} . }
\end{aligned}
$$

On dividing this by $\tau^{2}-1-2 \mathrm{~m}=P_{q}+P_{2}$ no accuracy is lost since $P_{q}$ or $\mathrm{m}^{2}$ are present explicitly.

$2^{\circ}$. Suppose $\tau=-1-\mathrm{m}+P_{q^{\circ}}$ Equate to zero the coefficient of $\zeta^{1+\tau}$. Then $a$ becomes of the same form as before and consequently no accuracy is lost on dividing by $\tau^{2}-1-2 \mathrm{~m}$.

The existence of this theorem is due to the fact that in $z$ we have the coefficients of $\zeta^{ \pm \tau}$ numerically equal and that there are two different sets of equations to determine them, since the equation $(3 e)$ is not symmetrical with respect to $\zeta, \zeta^{-1}$. We might have obtained another equation, $\left(3 e^{\prime}\right)$, in which $s,-D$ replace $u, D$ respectively. The sum and difference of $(3 e),\left(3 e^{\prime}\right)$ correspond to the rates of change of the areal velocity in the planes of $x z, y z$.

\section{Section III. Transformations of the Differential equations.}

17. Transformation to avoid the loss of accuracy when $\tau=P_{q}(q=1$ or 2$)$. The equations (1) are, for terms of order $\mu$,

$$
\begin{gathered}
\left(D^{2}+2 \mathrm{~m} D\right) u_{\mu}=\left[\frac{\partial f}{\partial s}\right]_{\mu}, \quad\left(D^{2}-2 \mathrm{~m} D\right) s_{\mu}=\left[\frac{\partial f}{\partial u}\right]_{\mu}, \\
D^{2} z_{\mu}=\frac{1}{2}\left[\frac{\partial f}{\partial z}\right]_{\mu}
\end{gathered}
$$

Multiply these by $s_{\nu}, u_{\nu}, 2 z_{\nu}$ and sum for all values of $\mu, \nu$ except $\mu=0$, $\nu=0$, such that

The result may be written

$$
\mu+\nu=\lambda
$$

$$
\begin{aligned}
& \sum_{\mu=1}^{\lambda-1}\left\{D^{2}\left(u_{\mu} s_{\nu}+z_{\mu} z_{\nu}\right)-2 D u_{\mu} \cdot D s_{\nu}-2 D z_{\mu} \cdot D z_{\nu}+2 \mathrm{~m}\left(s_{\mu} D u_{\nu}-u_{\mu} D s_{\nu}\right)\right\} \\
& =\sum_{\mu=1}^{\lambda-1}\left\{u_{\mu}\left[\frac{\partial f}{\partial u}\right]_{\nu}+s_{\mu}\left[\frac{\partial f}{\partial s}\right]_{\nu}+z_{\mu}\left[\frac{\partial f}{\partial z}\right]_{\nu}\right\} \\
& =\sum_{p^{\prime}=2}^{\lambda} \frac{1}{\left(p^{\prime}-1\right) !}\left[\left\{\left(u-u_{0}\right)\left[\frac{\partial}{\partial u}\right]_{0}+\left(s-s_{0}\right)\left[\frac{\partial}{\partial s}\right]_{0}+z\left[\frac{\partial}{\partial z}\right]_{0}^{p^{\prime}} f\right]_{\lambda}^{\prime}\right. \\
& =\sum_{p^{\prime}=2}^{\lambda} \frac{1}{\left(p^{\prime}-1\right) !}\left[\partial^{p^{\prime}} f\right]_{\lambda}^{\prime},
\end{aligned}
$$

in symbolic notation.*

* Thus the series is

$$
\left[\left(u-u_{0}\right)^{2}\left[\frac{\partial^{2} f}{\partial u^{2}}\right]_{0}+2\left(u-u_{0}\right)\left(s-s_{0}\right)\left[\frac{\partial^{2} f}{\partial u \partial s}\right]_{0}+\cdots+z^{2}\left[\frac{\partial^{2} f}{\partial z^{2}}\right]_{0}+\cdots\right]_{\lambda}^{\prime},
$$

where we put $u=u_{0}, s=s_{0}, z=0$ in the partial derivatives after they have been formed. 
Next, considering terms of order $\lambda$, equation $(2 B)$ may be written

$$
\begin{aligned}
D u_{0} \cdot D s_{\lambda}+D s_{0} \cdot D u_{\lambda}-u_{\lambda}\left[\frac{\partial f}{\partial u}\right]_{0} & -s_{\lambda}\left[\frac{\partial f}{\partial s}\right]_{0}+\sum_{\mu=1}^{\lambda-1}\left(D u_{\mu} \cdot D s_{\nu}+D z_{\mu} \cdot D z_{\nu}\right) \\
& =\sum_{p^{\prime}=2}^{\lambda} \frac{1}{p^{\prime} !}\left[\partial^{p^{\prime}} f\right]_{\lambda}^{\prime}+C-\left[D^{-1}\left(D^{\prime} f\right)\right]_{\lambda}^{\prime},
\end{aligned}
$$

in which the known and unknown terms have been separated.

Divide the former of these equations by 2 and subtract from the latter so as to eliminate the terms in $\partial^{2} f$. Also transform the partial derivatives of the first line of (8) by means of (6) with $\mu=0$. The result may be written

$$
D u_{0} \cdot D s_{\lambda}+D s_{0} \cdot D u_{\lambda}-\left(u_{\lambda} D^{2} s_{0}+s_{\lambda} D^{2} u_{0}\right)+2 \mathrm{~m}\left(u_{\lambda} D s_{0}-s_{\lambda} D u_{0}\right)
$$

(9) $+\sum_{\mu=1}^{\lambda-1}\left\{-\frac{1}{2} D^{2}\left(u_{\mu} s_{\nu}+z_{\mu} z_{\nu}\right)+2\left(D u_{\mu} \cdot D s_{\nu}+D z_{\mu} \cdot D z_{\nu}\right)+\mathbf{m}\left(u_{\mu} D s_{\nu}-s_{\mu} D u_{\nu}\right)\right\}$

$$
=\sum_{p^{\prime}=3}^{\lambda} \frac{p^{\prime}-2}{2\left(p^{\prime} !\right)}\left[\partial^{p^{\prime}} f\right]_{\lambda}^{\prime}+C-\left[D^{-1}\left(D^{\prime} f\right)\right]_{\lambda}^{\prime} \text {. }
$$

Also, equation $(2 d)$ may be written

$$
\begin{aligned}
& D^{2}\left(u_{0} s_{\lambda}+s_{0} u_{\lambda}\right)-\left(D u_{0} \cdot D s_{\lambda}\right.\left.+D s_{0} \cdot D u_{\lambda}\right) \\
&+2 \mathrm{~m}\left(s_{0} D u_{\lambda}-u_{0} D s_{\lambda}+s_{\lambda} D u_{0}-u_{\lambda} D s_{0}\right) \\
&(10)+\sum_{\mu=1}^{\lambda-1}\left\{D^{2}\left(u_{\mu} s_{\nu}+z_{\mu} z_{\nu}\right)-\left(D u_{\mu} \cdot D s_{\nu}+D z_{\mu} \cdot D z_{\nu}\right)+2 \mathrm{~m}\left(s_{\mu} D u_{\nu}-u_{\mu} D s_{\nu}\right)\right\} \\
&=\sum_{p=2}^{\infty}\left[(p+1) f_{p}\right]_{\lambda}+C-\left[D^{-1}\left(D^{\prime} f\right)\right]_{\lambda}^{\prime} .
\end{aligned}
$$

The terms of principal importance for the determination of $\lambda, \lambda^{\prime}$ in (9), (10) are

$$
\begin{gathered}
-\tau\left(\lambda-\lambda^{\prime}\right)-2(1+\mathrm{m})\left(\lambda+\lambda^{\prime}\right), \\
\tau(1+2 \mathrm{~m})\left(\lambda-\lambda^{\prime}\right)+\left(\tau^{2}+1+4 \mathrm{~m}\right)\left(\lambda+\lambda^{\prime}\right) .
\end{gathered}
$$

Now $\tau^{2}=P_{2 q}$ when $\tau=P_{q}$. Hence, multiplying (9) by $(1+3 \mathrm{~m}),(10)$ by 2 and adding, we obtain an equation for the determination of $\tau\left(\dot{\lambda}-\lambda^{\prime}\right)$ which, when divided by $\tau$, i. e., subjected to the operation $D^{-1}$, will give $\lambda-\lambda^{\prime}$. The equation which results from these processes is, after one or two transformations,

$$
\begin{gathered}
2 \tau\left(u_{\lambda} s_{0}+s_{\lambda} u_{0}\right)-\left(u_{\lambda} D s_{0}+s_{\lambda} D u_{0}\right)(1-3 \mathrm{~m})+4 \mathrm{~m}\left(u_{\lambda} s_{0}-s_{\lambda} u_{0}\right) \\
-6(\mathrm{~m} / \tau)\left\{u_{\lambda}\left(D^{2} s_{0}+D s_{0}-\mathrm{m} D s_{0}\right)+s_{\lambda}\left(D^{2} u_{0}-D u_{0}+\mathrm{m} D u_{0}\right)\right\} \\
(11)+3 \sum_{\mu=1}^{\lambda-1}\left\{\frac{1}{2}(1-\mathrm{m}) \tau\left(u_{\mu} s_{\nu}+z_{\mu} z_{\nu}\right)+(\mathrm{m} / \tau)\left\{2\left(D u_{\mu} D s_{\nu}+D z_{\mu} \cdot D z_{\nu}\right)\right.\right. \\
\left.\left.+(1-\mathrm{m})\left(s_{\mu} D u_{\nu}-u_{\mu} D s_{\nu}\right)\right\}\right\} \\
=\frac{2}{\tau} \sum_{p=2}^{\infty}\left[(p+1) f_{p}\right]_{\lambda}+\frac{1+3 \mathrm{~m}}{\tau} \sum_{p^{\prime}=3}^{\lambda} \frac{p^{\prime}-2}{2\left(p^{\prime} !\right)}\left[\partial^{p^{\prime}} f\right]_{\lambda}^{\prime}-\frac{3(1+\mathrm{m})}{\tau^{2}}\left[D^{\prime} f\right]_{\lambda}^{\prime} .
\end{gathered}
$$


For inequalities of the first two orders in which $\tau=P_{1}$ (there are none for $\tau=P_{2}$ ), this equation serves to prevent any loss of accuracy. For in the left hand member, $\tau$ only appears as a divisor in the combination $\mathrm{m} / \tau$. The first term of the right hand member has the combination $\mathrm{m}^{2} / \tau$, the second term is absent, the third term has the combination $\mathbf{m}^{3} / \tau^{2}$.

When $\tau=P_{2}$, the only terms in the left hand member which give rise to a loss of accuracy are those multiplied by $\mathrm{m} / \tau$. For the first of these it should be noted that $D^{2} s_{0}+D s_{0}, D^{2} u_{0}-D u_{0}$ are divisible by $\mathrm{m}^{2}$.

18. Transformation to avoid the loss of accuracy when $\pm \tau=\mathrm{c}+P_{2}$ and the characteristic contains $\mathbf{k}^{2}$.

We have the equations*

$$
\begin{aligned}
\left(D^{2}+2 \mathrm{~m} D\right) u_{\mathrm{b}} & =\left[\frac{\partial f}{\partial s}\right]_{\mathrm{b}}, & \left(D^{2}-2 \mathrm{~m} D\right) s_{\mathrm{b}} & =\left[\frac{\partial f}{\partial u}\right]_{\mathrm{b}}, \\
\left(D^{2}+2 \mathrm{~m} D\right) u_{\mathrm{k}^{2}} & =\left[\frac{\partial f}{\partial s}\right]_{\mathrm{k}^{2}}, & \left(D^{2}-2 \mathrm{~m} D\right) s_{\mathrm{k}^{2}} & =\left[\frac{\partial f}{\partial u}\right]_{\mathrm{k}^{2}} \\
D^{2} z_{\mathrm{k}} & =\frac{1}{2}\left[\frac{\partial f}{\partial z}\right]_{\mathrm{k}}, & D^{2} z_{\mathrm{kb}} & =\frac{1}{2}\left[\frac{\partial f}{\partial z}\right]_{\mathrm{kb}}
\end{aligned}
$$

in which $b$ is a characteristic of order $\lambda-2$ and not containing $k$. The right hand members of these equations may be expanded in powers of

$$
u-u_{0}, s-s_{0}, z,
$$

the first pair does not contain $z$, the second pair contains $z$ only in the second power, and the third pair only in the first power. The right hand members may be therefore written

$$
\begin{gathered}
u_{\mathrm{b}}\left[\frac{\partial^{2} f}{\partial s \partial u}\right]_{0}+s_{\mathrm{b}}\left[\frac{\partial^{2} f}{\partial s^{2}}\right]_{0}+\left[\frac{\partial f}{\partial s}\right]_{\mathrm{b}}^{\prime}, \quad u_{\mathrm{b}}\left[\frac{\partial^{2} f}{\partial u^{2}}\right]_{0}+s_{\mathrm{b}}\left[\frac{\partial^{2} f}{\partial u \partial s}\right]_{0}+\left[\frac{\partial \dot{f}}{\partial u}\right]_{\mathrm{b}}^{\prime}, \\
u_{\mathbf{k}^{2}}\left[\frac{\partial^{2} f}{\partial s \partial u}\right]_{0}+s_{\mathrm{k}^{2}}\left[\frac{\partial^{2} f}{\partial s^{2}}\right]_{0}+\frac{1}{2} z_{\mathrm{k}}^{2}\left[\frac{\partial^{3} f}{\partial s \partial z^{2}}\right]_{0}, \\
u_{\mathbf{k}^{2}}\left[\frac{\partial^{2} f}{\partial u^{2}}\right]_{0}+s_{\mathrm{k}^{2}}\left[\frac{\partial^{2} f}{\partial s \partial u}\right]_{0}+\frac{1}{2} z_{\mathrm{k}}^{2}\left[\frac{\partial^{3} f}{\partial u \partial z^{2}}\right]_{0}, \\
\frac{1}{2} z_{\mathrm{k}}\left[\frac{\partial^{2} f}{\partial z^{2}}\right]_{0}, \quad \frac{1}{2} z_{\mathrm{kb}}\left[\frac{\partial^{2} f}{\partial z^{2}}\right]_{0}+\frac{1}{2} z_{\mathrm{k}} u_{\mathrm{b}}\left[\frac{\partial^{3} f}{\partial u \partial z^{2}}\right]_{0}+\frac{1}{2} z_{\mathrm{k}} s_{\mathrm{b}}\left[\frac{\partial^{3} f}{\partial s \partial z^{2}}\right]_{0}+\frac{1}{2}\left[\frac{\partial f}{\partial z}\right]_{\mathrm{kb}}^{\prime},
\end{gathered}
$$

where the functions []$_{\mathrm{b}}^{\prime},[]_{\mathrm{kb}}^{\prime}$ do not contain $u_{\mathrm{b}}, s_{\mathrm{b}}, z_{\mathrm{kb}}$, and are at least of the second degree with reference to $u-u_{0}, s-s_{0}, z$ or contain the factor $\mathrm{m}^{2}$. If, then,

* In these equations it is more convenient to specify the characteristic than its order; both methods are used in this article. 
we multiply the six equations by $s_{\mathrm{k}^{2}}, u_{\mathrm{k}^{2}},-s_{\mathrm{b}},-u_{\mathrm{b}},-z_{\mathrm{kb}}, z_{\mathrm{k}}$ respectively, all the terms but those arising from []$_{\mathrm{b}}^{\prime},[]_{\mathrm{kb}}^{\prime}$ will vanish and we obtain

$$
\begin{aligned}
D\left\{s_{\mathbf{k}^{2}} D u_{\mathrm{b}}+u_{\mathbf{k}^{2}} D s_{\mathrm{b}}-s_{\mathrm{b}} D\right. & u_{\mathbf{k}^{2}}-u_{\mathrm{b}} D s_{\mathbf{k}^{2}}+2 \mathrm{~m}\left(s_{\mathbf{k}^{2}} u_{\mathrm{b}}-u_{\mathbf{k}^{2}} s_{\mathrm{b}}\right) \\
& \left.+z_{\mathbf{k}} D z_{\mathbf{k b}}-z_{\mathbf{k b}} D z_{\mathbf{k}}\right\}-\left[Q_{3}+\mathbf{m}^{2} Q_{2}\right]_{\mathbf{k}^{2} \mathrm{~b}}^{\prime}=0,
\end{aligned}
$$

where $Q_{3}, Q_{2}$ are power series in $u-u_{0}, s-s_{0}, z$ containing only even powers of $z$ and not containing $u_{b}, s_{b}, z_{\mathrm{kb}}$, the first terms being of degrees 3,2 respectively.

For the terms of characteristic $b^{2}$ which have the small divisor $\pm \tau-\mathrm{c}=P_{q}$ we have to consider the expression (5)' . The portion of this containing $u_{\mathbf{y}^{3}}$, $s_{\mathrm{k}^{2}}, z_{\mathrm{kb}}$ is

$$
\begin{aligned}
& -D\left(u_{\mathbf{k}^{2}} \zeta^{-1}\right) \cdot D\left(s_{\mathrm{b}} \zeta\right)-D\left(u_{\mathrm{b}} \zeta^{-1}\right) \cdot D\left(s_{\mathbf{k}^{2}} \zeta\right) \\
& \quad+2\left(z_{\mathrm{k}} D^{2} z_{\mathrm{kb}}+z_{\mathrm{kb}} D^{2} z_{\mathrm{k}}+D z_{\mathrm{k}} \cdot D_{z_{\mathrm{kb}}}\right) .
\end{aligned}
$$

Adding twice the left-hand member of (12) to this, it becomes

$$
\begin{aligned}
& -D\left(u_{\mathbf{k}^{2}} \zeta^{-1}\right) \cdot D\left(s_{\mathrm{b}} \zeta\right)-D\left(u_{\mathrm{b}} \zeta^{-1}\right) \cdot D\left(s_{\mathbf{k}^{2}} \zeta\right) \\
& +2 D\left(s_{\mathbf{k}^{2}} D u_{\mathrm{b}}+u_{\mathbf{k}^{2}} D s_{\mathrm{b}}-s_{\mathrm{b}} D u_{\mathbf{k}^{2}}-u_{\mathrm{b}} D s_{\mathbf{k}^{2}}\right) \\
& +2 \mathrm{~m} D\left(s_{\mathbf{k}^{2}} u_{\mathrm{b}}-u_{\mathbf{k}^{2}} s_{\mathrm{b}}\right)+4 z_{\mathrm{k}} D^{2} z_{\mathrm{kb}}+2 D z_{\mathrm{k}} \cdot D z_{\mathrm{kb}}-2\left[Q_{3}+\mathrm{m}^{2} Q_{2}\right]_{\mathrm{k}^{2} \mathrm{~b}} \cdot
\end{aligned}
$$

Since the coefficients of $\zeta^{ \pm \tau}$ in this expression are equal, it is sufficient to consider the coefficients of $\zeta^{\tau}$.

First, suppose that $\tau-2 \mathrm{~g}$ does not contain $\mathrm{g}$. We have

$$
u_{\mathbf{k}^{2}} \zeta^{-1}=\left(k^{2}\right)_{0} \zeta^{2 g}+\left({k^{\prime 2}}^{2}\right)_{0} \zeta^{-2 g}+(1,10),
$$

where $\left(k^{2}\right)_{0},\left(k^{\prime 2}\right)_{0}$ are known to $\mathrm{m}^{10}$ (Art. 22), and suppose that

$$
u_{\mathrm{b}} \zeta^{-1}=\beta \zeta^{\sigma}+\beta^{\prime} \zeta^{-\sigma}+(1,10) \text {, }
$$

in which $\sigma+2 \mathrm{~g}=\tau$. Substituting in (13), we obtain for the coefficient of $\beta\left(k^{\prime 2}\right)_{0} \zeta^{\tau}$,

$$
-2 \sigma \mathrm{g}+2(\sigma+2 \mathrm{~g})(\sigma-2 \mathrm{~g}+2 \mathrm{~m}+2)=2 \sigma(\tau-\mathrm{g})+(2,11)=(q, 11),
$$

since (Art. 20) $\mathrm{g}=1+\mathrm{m}+(2,11), \tau-\mathrm{g}=\tau-\mathrm{c}-(\mathrm{g}-\mathrm{c})=(q, 11)$, when $\tau-\mathrm{c}=P_{q}$. The coefficient of $\left(k^{2}\right)_{0} \beta^{\prime} \zeta^{\tau}$ is not small. But $\left(k^{2}\right)_{0}=(2,11)$. For, if we put $\tau=2 \mathrm{~g}=2(1+\mathrm{m})+(2,11), \lambda=\left(k^{2}\right)_{0}, \lambda^{\prime}=\left(k^{\prime 2}\right)_{0}$ in $(3 a)$ this expression becomes

$$
-(4-4 \mathrm{~m})\left(k^{2}\right)_{0}+\left({k^{\prime}}^{2}\right)_{0} P_{2} \text {. }
$$

All the other terms in $(2 a)$ either contain the factor $\mathrm{m}^{2}$ explicitly or are formed from products $(1,10)(2,10)$ and are, therefore, at least equal to $(2,11)$. Trans. Am. Muth. Soc. 12 
Also $\left(k^{\prime 2}\right)_{0}=(0,10)$ and, therefore, $\left(k^{\prime 2}\right)_{0} P_{2}=(2,11)$; as there is no small divisor we obtain $\left(k^{2}\right)_{0}=(2,11)$.

Again, we have

$$
z_{\mathrm{k}} \sqrt{-1}=k_{0}\left(\zeta^{\mathrm{g}}-\zeta^{-\mathrm{g}}\right)+(1,10)
$$

where $k_{0}=(0,10)$ (Art. 31), and suppose that

$$
z_{\mathrm{kb}} \sqrt{-1}=\gamma\left(\zeta^{\tau-\mathrm{g}}-\zeta^{-\tau+\mathrm{g}}\right)+(1,10) .
$$

Then the term involving $\gamma k_{0} \zeta^{\tau}$ in (13) has the coefficient $\tau-\mathrm{g}=(q, 11)$ and all others terms arising from $z_{\mathrm{kb}}$ are equal to $(1,10)(1,10)=(2,11)$ at the worst.

Collecting these results we see that all the terms of (13) except those in $Q_{3}$ are either multiplied by $\mathrm{m}^{2}$, or are obtained to $\mathrm{m}^{11}$ or consist of the expressions $\beta(2,11), \beta^{\prime}(2,11)$ - the result which this investigation was undertaken to demonstrate.

Second, suppose that $\tau$ does not contain $2 \mathrm{~g}$. Then

$$
u_{\mathrm{k}^{2}} \zeta^{-1}=\left(k k^{\prime}\right)_{0} \zeta^{0}+(1,10),
$$

where $\left(k k^{\prime}\right)_{0}=(0,10)$. We use the untransformed expression $\left(5^{\prime}\right)$. The first two terms then contain the factor $(2,10)$ at least and it is only necessary to examine $(D z)^{2}-D^{2}\left(z^{2}\right)$.

\section{Section IV. The Degrees of aCcuracy of the coefficients.}

19. The intermediate orbit is the variational curve. It will be assumed that the coefficients of the periodic terms in this orbit are obtained to a given degree of accuracy (Art. 4) equal to 10, and we shall examine the degrees of accuracy to which the terms of the first, second, ... orders may be found. I use a special number instead of a general one; nothing is lost in generality by this, for if we require the degrees of accuracy for any other starting number $p$, all that is necessary is to add $p-10$ to the degrees of accuracy throughout. The special number enables me to set down the results more briefly and clearly, and, moreover, by Art. 5, they are ready for immediate practical application.

The argument refers throughout to the power of $\zeta$ in $u \zeta^{-1}, z \sqrt{-1}$, unless otherwise stated.

It will frequently occur that coefficients may be found more accurately and may begin with a higher power of $m$ than the statement shows. In all cases, the statement refers to the lowest degree of accuracy and the lowest power of $\mathbf{m}$ which it is necessary to consider; that is, the expression $(p, q)$ may include the cases $\left(p+p^{\prime}, q+q^{\prime}\right)$ where $p^{\prime} \geqq 0, q^{\prime} \geqq 0$, except in the cases of divisors when $p^{\prime}$ is always zero and $q+q^{\prime}$ is either 11 or $\infty$. See Art. 7 (v).

The results are all collected in the table of Art. 31 ; the discussion will be more easily followed by referring to this table rather than to the text. 
20. Here $u=u_{0}$ and

Terms of Zero Order.

$$
u_{0} \zeta^{-1}=\sum_{i} a_{i} \zeta^{2 i} \quad(i=0, \pm 1, \pm 2, \cdots) .
$$

As $a_{0}$ is the unit of length, we shall put $a_{0}=1$. Then

$$
a_{0}=(0, \infty), \quad a_{i}=(2 i, 10)=(2,10)
$$

The principal parts of $\mathrm{c}, \mathrm{g}$ can be found to $\mathrm{m}^{11}$ (Art. 4), so that

$$
\mathrm{e}=1+\mathrm{m}+(2,11), \mathrm{g}=1+\mathrm{m}+(2,11) .
$$

Also the terms containing $\mathbf{m}^{0}, \mathrm{~m}^{\mathbf{1}}, \mathrm{m}^{2}$ never all disappear at the same time from $i+i^{\prime} \mathrm{c}+i^{\prime \prime} \mathrm{g}+i^{\prime \prime \prime} \mathrm{m}$, i. e., from any divisor, to the degree of approximation considered here (Art. 3).

\section{Terms of the First Order.}

21. There are terms for which $\tau=P_{q}, \tau-\mathrm{c}=P_{q}$, where $q=1$, but none for which $q=2$. For such terms the expressions $(4),(5)^{\prime}$ respectively reduce to

$$
\begin{gathered}
-D u_{1} \cdot D s_{0}-D s_{1} \cdot D u_{0}+\frac{1}{2}\left(u_{1} D s_{0}-s_{1} D u_{0}+u_{0} D s_{1}-s_{0} D u_{1}\right), \\
-D\left(u_{1} \zeta^{-1}\right) \cdot D\left(s_{0} \zeta\right)-D\left(u_{0} \zeta^{-1}\right) \cdot D\left(s_{1} \zeta\right) .
\end{gathered}
$$

Since $D\left(u_{0} \zeta^{-1}\right), D\left(s_{0} \zeta\right)$ are equal to $(2,10)$, these may be written

$$
\begin{array}{rr}
\frac{1}{2} D\left(u_{1} s_{0}-s_{1} u_{0}\right)+(2,10) D u_{1}+(2,10) D s_{1} & \left(\tau=P_{1}\right) \\
(2,10) D\left(u_{1} \zeta^{-1}\right)+(2,10) D\left(s_{1} \zeta\right) & \left(\tau-\mathrm{c}=P_{1}\right) .
\end{array}
$$

For the characteristic $e^{\prime}$, the coefficients of $\zeta^{ \pm \mathrm{m}}$ have the divisor $\mathrm{m}$ and therefore all coefficients can be obtained to $\mathrm{m}^{9}$. But $(4)_{1}$, on division by $m$, gives, for terms other that the principal ones, $(1,9)(2,10) \div m=(2,10)$, and all the other coefficients can be obtained to $\mathrm{m}^{10}$. Noting $(3 a),(2 d)$ we find that

$$
\eta_{0}, \eta_{0}^{\prime}=(1,10) ; \quad \eta_{0}+\eta_{0}^{\prime}, \eta_{i}, \eta_{i}^{\prime}=(2,10)
$$

For the characteristic $a$, the coefficients of $\zeta^{ \pm 1}$ have the divisor

$$
1-\mathrm{c}=(1,11) \text {. }
$$

Treating these coefficients in a similar manner with $(5)_{1},(3 a),(2 d)$ we obtain

$$
a_{ \pm 1 / 2}=(1,10) ; \quad 3 a_{1 / 2}+a_{-1 / 2}, a_{i}=(2,10) \quad\left(i \neq \pm \frac{1}{2}\right) .
$$

For the characteristic e, there is more difficulty in avoiding loss of accuracy. We put

$$
u_{\theta} \zeta^{-1}=\sum_{i}\left(\epsilon_{i} \zeta^{\mathrm{c}+2 i}+\epsilon_{-i}^{\prime} \zeta^{-\mathrm{c}-2 i}\right) \quad(i=0, \pm 1, \cdots)
$$


and use equations $(2 a),(2 f)$. One of the pair $\epsilon_{0}, \epsilon_{0}^{\prime}$ is arbitrary; put*

$$
\epsilon_{0}-\epsilon_{0}^{\prime}=1 \text {. }
$$

The equation will then give $\epsilon_{-1}, \epsilon_{1}^{\prime}=(1,9), \epsilon_{-1}+3 \epsilon_{1}^{\prime}=(2,10)$, and all the other coefficients with a degree of accuracy 10 .

But adding equations $(2 B),(2 d)$, we find, for the terms giving $u_{e}$,

$$
\begin{aligned}
D^{2}\left(u_{\mathrm{e}} s_{0}+s_{\mathrm{e}} u_{0}\right) & =[f]_{\mathrm{e}}=u_{\mathrm{e}}\left[\frac{\partial f_{-1}}{\partial u}\right]_{0}+s_{\mathrm{e}}\left[\frac{\partial f_{-1}}{\partial s}\right]_{0}+3\left[f_{2}^{\prime}\right]_{\mathrm{e}} \\
& =\left(u_{\mathrm{e}} s_{0}+s_{\mathrm{e}} u_{0}\right) \frac{\kappa}{r_{0}^{3}}+\mathrm{m}^{2}(0,9)
\end{aligned}
$$

from the values already found. Since $\kappa / r_{0}^{3}=1+2 m+(2,10)$, we have for the coefficient of $\zeta^{\mathrm{c}-2}$ in this equation,

$$
\left\{(\mathrm{c}-2)^{2}-1-2 \mathrm{~m}+(2,10)\right\}\left\{\epsilon_{-1}+\epsilon_{1}^{\prime}+(2,10)\right\}=(2,11),
$$

or, since $\epsilon_{-1}+\epsilon_{1}^{\prime}=(1,9),(\mathrm{c}-2)^{2}-1-2 \mathrm{~m}=(1,11)$,

$$
(1,11)\left(\epsilon_{-1}+\epsilon_{1}^{\prime}\right)+(1,9)(2,10)=(2,11) .
$$

Hence $\epsilon_{-1}+\epsilon_{1}^{\prime}=(2,11) \div(1,11)=(1,10)$. This, with $\epsilon_{-1}+3 \epsilon_{1}^{\prime}=(2,10)$, gives the following results:

$$
\begin{aligned}
& \epsilon_{0}, \epsilon_{0}^{\prime}=(0,11) ; \quad \epsilon_{-1}, \epsilon_{1}^{\prime}=(1,10) ; \\
& 3 \epsilon_{0}+\epsilon_{0}^{\prime}, \epsilon_{-1}+3 \epsilon_{1}^{\prime}, \epsilon_{i}^{\prime}=(2,10) \quad(i \neq 0,-1) \text {. }
\end{aligned}
$$

The coefficients of $z_{\mathrm{k}}$ are given in the table.

\section{Terms of the Second Order.}

22. For the terms for which $\tau=P_{1}$ we can use equation (11) which determines all such coefficients without loss of accuracy, since the second term of the right-hand member does not exist.

There are no terms for which $\tau=P_{2}$.

The terms for which $\tau-\mathrm{c}=P_{1}$ or $P_{2}$ can only occur with the characteristics $\mathrm{e} e^{\prime}, e^{\prime} a$.

The terms with characteristic $e e^{\prime}$ are formed of the products of terms with characteristics $0, \mathrm{e} e^{\prime}$ and $\mathrm{e}, e^{\prime}$; they all contain $\mathrm{m}$ as a factor and the small divisors are equal to $P_{1}=(1,11)$. Now the characteristic $e^{\prime}$ only occurs in $\left(5^{\prime}\right)$ in the forms $D\left(u_{a^{\prime}} \zeta^{-1}\right), D\left(s_{e^{\prime}} \zeta\right)$, and these are equal to $\mathrm{m}(1,10)=(2,11)$ for the coefficients of $\zeta^{ \pm m}$. When the multiplication is made with the terms of characteristic e, we obtain for the coefficients of $\zeta^{ \pm \pm m}$

\footnotetext{
*T. M. M., pt. I, p. 71.
} 


$$
(2,11)(0,10)+(2,10)(1,10)=(2,11) ;
$$

this, divided by $P_{1}$, gives $(1,10)$. The terms formed by combining the terms of characteristics $0, \mathrm{e} e^{\prime}$ are at the worst equal to $(2,10)(1,10)=(3,11)$, which, divided by $(1,11)$, gives $(2,10)$. Hence all are obtained accurately to $\mathrm{m}^{10}$.

The terms with characteristic $e^{\prime} a$ are treated in the same way. The terms with arguments $\pm(1-\mathrm{m})$ which give a divisor $P_{1}$ contain the factor $\mathrm{m}$, those with arguments $\pm(1+\mathrm{m})$ which give a divisor $P_{2}$ do not contain this factor. For those formed of the products from $e^{\prime}$ and $a$ we obtain $(2,10)(1,10)=(3,11)$. Considering the combinations $0, e^{\prime} a$, we find that for arguments $\pm(1-\mathbf{m})$, $(5)^{\prime}$ is equal to $(0,9)(2,10)$, which, divided by $P_{1}$, gives $(1,9)$, and that for arguments $\pm(1+\mathrm{m}),(5)^{\prime}$ is equal to $(1,9)(2,10)$, which, divided by $P_{2}$, gives $(1,9)$. The remaining coefficients which have no small divisors can be found to $\mathrm{m}^{10}$. Also

$$
3(\eta a)_{1 / 6}+\left(\eta^{\prime} a\right)_{-3 / 2}=(2,10), \quad(\eta a)_{-1 / 2}+3\left(\eta^{\prime} a\right)_{1 / 2}=(2 ; 10) .
$$

It is necessary for the third order terms to obtain $\left(\eta^{2}\right)_{1},\left({\eta^{\prime 2}}^{2}\right)_{-1}$ to $\mathrm{m}^{11}$. That this is possible is seen by substituting $\tau= \pm 2(1+\mathrm{m})$ successively in $(3 a)$; the terms of principal importance becomes $-4(1+\mathrm{m}) \lambda^{\prime},-4(1+\mathrm{m}) \lambda$. All the other terms in $(2 a)$ are of the form $(2,10)(1,10)=(3,11)$ or are factored by $\mathrm{m}$ or $\mathrm{m}^{2}$ and there is no small divisor.

All terms in $z$ are obtained to $\mathrm{m}^{10}$.

Thus all inequalities of the second order are obtained to $\mathrm{m}^{10}$ except those with characteristic $e^{\prime} a$ and arguments $\pm 1 \pm \mathrm{m}$; these are obtained to $\mathrm{m}^{9}$.

\section{Terms of the Third Order.}

23. It will be shown that all but two coefficients can be found to $\mathrm{m}^{9}$.

Consider the equations $(3 a),(3 f)$ for coefficients which have no small divisors. We shall show presently that in the worst case of small divisors the coefficients can be obtained to $\mathrm{m}^{8}$. In the equations for coefficients with no small divisors, the coefficients with small divisors are multiplied by $(2,10)$ at least. Hence no loss of accuracy arises from this source. The only terms of lower orders which are not obtained to $\mathrm{m}^{10}$ are the coefficients of $\zeta^{ \pm 1 \pm m}$ in the characteristic $e^{\prime} a$. As third order terms are formed in these equations from products of first and second order terms, these products in this particular case will be

$$
(p, 10)(0,9)=(1,10) \text { unless } p=0,
$$

and $p=0$ only for the coefficients of $\zeta^{ \pm 0}$. Hence the only coefficients which are not obtained to $\mathrm{m}^{10}$ are those of characteristic e $e^{\prime} a$ and arguments $\pm \mathrm{c} \pm 1 \pm \mathrm{m}$; of these, the arguments $\pm(\mathrm{c}+1 \pm \mathrm{m})$ do not give rise to small divisors. 
24. For the case $\tau=P_{1}$, the equation (11) is sufficient to avoid all loss of accuracy, except for the second term in the right-hand member. But this, being of the third degree with respect to powers and products of $u-u_{0}, s-s_{0}, z$ must be formed of first order terms only. These are all equal to $(1,10)$ at least except for the characteristics $\mathrm{e}^{3}, \mathrm{ek}^{2}$, which do not give rise to small divisors $\tau=P_{1}$. On division by $\tau=P_{1}$ the results are in any case true to $\mathrm{m}^{9}$.

The only case in which $\tau=P_{2}$ is that of the coefficients of $\zeta^{ \pm(c-1-\mathrm{m})}$ in the characteristic e $e^{\prime} a$. The only term in which we appear to lose more than one degree of accuracy is the second term in the right-hand member of (11). But this term is formed from the characteristics e, $e^{\prime}, a$, that is, it is of the order

$$
(0,10)(1,10)(1,10) \div(2,11)=(1,9),
$$

at the worst. The first term involving $\mathrm{m} / \tau$ in the right-hand member is, at the worst, equal to

$$
\mathrm{m}\{(0,9)(2,10)+\mathrm{m}(0,9)\} \div(2,11)=(0,9) .
$$

The last term of the right-hand member involves a loss of one power of $\mathrm{m}$ but it does not contain terms of characteristic $e^{\prime} a$ and therefore can be formed to $\mathrm{m}^{9}$; it contains a term involving $\mathrm{m}^{-1}$. This term is cancelled by a similar term in the second term of the left-hand member factored by $\mathrm{m} / \tau$, but as this term contains terms from the characteristic $e^{\prime} a$ it can only be found to $\mathrm{m}^{8}$.* $^{*}$

25. When $\tau=\mathrm{c}+P_{q}$, the only cases in which we might not be able to obtain the terms correctly to $\mathrm{m}^{9}$ occur in the characteristics $e^{\prime 2} a, \mathrm{ek}^{2}, \mathrm{e} e^{\prime 2}$.

For the only case in which the terms of the first and second orders are not known to $\mathrm{m}^{10}$ occur in the characteristic $e^{\prime} a$; but the expression $(5)^{\prime}$ shows that these exceptional terms occur only multiplied by terms of characteristic $e^{\prime}$, that is, they are of the form

$$
(2,10)(0,9) \div(1,11)=(1,9),
$$

since $D\left(u_{e^{\prime}} \zeta^{-1}\right), D\left(s_{e^{\prime}} \zeta\right)$ are equal to $(2,10)$ and $q=1$ for these terms. The products of terms of characteristics $e^{\prime} a$ with those of characteristics $e^{\prime}, a$ do not give arguments $\tau=\mathrm{c}+P_{q}$.

Also the only terms which contain a divisor $P_{2}$ occur in the characteristics $\mathrm{e}^{2}, \mathrm{e} e^{\prime 2}$, and the terms of lower orders from which these are formed are known to $\mathrm{m}^{10}$. Hence the terms for which $q=2$ can in any case be obtained to $\mathrm{m}^{8}$, as the expression (5)' shows; suppose these (the worst case) are equal to $(0,8)$. Then for the terms in which $q=1$, the only case in which we could not obtain

* The terms involving $\mathrm{m}^{-1}$ could doubtless be transformed so as to cancel one another before development. But $I$ have not thought it worth while to attempt this since this characteristic is very small compared to most of those of the third order. It is to be noted that $D^{\prime} f$ contains the factor $\mathrm{m}^{3}$, since $t$ occurs only in $f_{p}$ in the form $\zeta^{i_{2} \mathrm{~m}}$ where $p \geqq 2$. 
the results correctly to $\mathrm{m}^{9}$ is that which arises in $\left(5^{\prime}\right)$ from the combination of the terms in $\mathrm{e}^{2}, \mathrm{e} e^{\prime 2}$ which are equal to $(0,8)$ with those of order zero. But the latter are equal to $(2,10)$. Hence the worst case in $(5)^{\prime}$. is $(0,8)(2,10)$. As this, when divided by $(1,11)$, gives $(1,9)$, we are able to obtain the terms in $\mathrm{ek}^{2}, \mathrm{e} e^{\prime 2}$ for which $\tau=\mathrm{c}+P_{1}$ to $\mathrm{m}^{9}$.

The arguments of the terms of characteristic $\mathrm{ek}^{2}$ which are equal to $\pm \mathrm{c}+P_{2}$ are $\pm(\mathrm{c}-2 \mathrm{~g})$. The combination of characteristics in $(5)^{\prime}$ are 0 with $\mathrm{ek}^{2}, \mathrm{e}$ with $\mathrm{k}^{2}, \mathrm{k}$ with $\mathrm{ke}$. The worst case of 0 with $\mathrm{ek}^{2}$ is, since the terms of arguments $\pm(c-2 g)$ do not enter,

$$
(1,9)(2,10) \div(2 ; 11)=(1,9) \text {; }
$$

they are therefore correct to $\mathrm{m}^{9}$. We use Art. 18 for the other combinations, putting $\mathrm{b}=\mathrm{e}$. Then

$$
\beta, \beta^{\prime}=(0,10), \quad\left[Q_{3}\right]_{\mathrm{k}^{2} \mathrm{e}}^{\prime}=0,
$$

since $Q_{3}$ does not contain terms of characteristics e, $\mathrm{ek}^{2}, \mathrm{ek}$, and the terms with characteristics $k^{2}, k$ can only arise in combination with $e$, ke respectively. All the other conditions of Art. 18 are fulfilled and therefore the results can be obtained to $\mathrm{m}^{9}$.

Finally the arguments of the terms in e $e^{\prime 2}$ which are equal to $\pm \mathrm{c}+P_{2}$ are $\pm(2-\mathrm{c}+2 \mathrm{~m})$. The combinations of characteristics in $\left(5^{\prime}\right)$ are $e^{\prime}, \mathrm{e} e^{\prime}$ and $e, e^{\prime 2}$. The former gives $(1,10)(1,10)=(2,11)$. The only cases in the latter which are not also equal to the same, are the combinations of $\epsilon_{0}$, $\epsilon_{0}^{\prime}$ with $\left(\eta^{2}\right)_{2},\left(\eta^{\prime 2}\right)_{-2}$. But we have obtained (Art. 22) the latter pair to $\mathrm{m}^{11}$ and therefore the combinations are $(0,10)(2,11)=(2,11)$. Hence, on division by $(2,11)$ all terms can be obtained to $\mathrm{m}^{9}$.

26. As there is no loss of accuracy in the equation for $z$ (Art. 15) the only terms in $z$ which cannot be obtained to $\mathrm{m}^{10}$ are those arising from $e^{\prime} a$ and therefore contained in the characteristic $e^{\prime} a$, with arguments $\pm \mathrm{g} \pm(1 \pm \mathrm{m})$; these are equal to $(0,9)$.

$$
\text { Let* } \quad \mu\left(\zeta^{\mathrm{g}+1+\mathrm{m}}-\zeta^{-\mathrm{g}-1-\mathrm{m}}\right)+\mu^{\prime}\left(\zeta^{\mathrm{g}-1-\mathrm{m}}-\zeta^{-\mathrm{g}+1+\mathrm{m}}\right)
$$

be the terms with argument $\pm \mathrm{g} \pm(1+\mathrm{m})$ in $z \sqrt{-1}$, and in equation $(2 e)$ separate out the terms which are not true to $\mathrm{m}^{10}$. We find

$$
D\left(u_{0} D z_{\mathrm{ke}^{\prime} a}-z_{\mathrm{ke}^{\prime} a} D u_{0}+u_{e^{\prime} a} D z_{\mathrm{k}}-z_{\mathrm{k}} D u_{e^{\prime} a}\right)=(1,10) \text {. }
$$

Equate to zero the coefficients of $\zeta^{g+1+m+1}, \zeta^{g-1-m+1}$ and isolate the terms which are not equal to $(1,10)$. We find

* So that, according to the general scheme of notation,

$$
\mu=(k \eta a)_{1 / 2}, \quad \mu^{\prime}=\left(k \eta^{\prime} a\right)_{-1 / 2}, \quad \mu_{1}=\left(k \eta^{\prime} a\right)_{1 / 2}, \quad \mu_{1}^{\prime}=(k \eta a)_{-1 / 2} .
$$


Whence

$$
3\left\{\mu-(\eta a)_{3 / 3} k_{0}\right\}=(1,10), \quad\left\{-\mu^{\prime}+\left(\eta^{\prime} a\right)_{-1 / 2} k_{0}\right\}=(1,10) .
$$

$$
\begin{aligned}
3 \mu+\mu^{\prime} & =k_{0}\left\{3(\eta a)_{1 / 2}+\left(\eta^{\prime} a\right)_{-\frac{1}{3}}\right\}+(1,10) \\
& =(0,10)(2,10)+(1,10)=(1,10),
\end{aligned}
$$

by the result at the end of Art. 22 .

Let $\mu_{1}, \mu_{1}^{\prime}$ be the coefficients for the arguments $\pm \mathrm{g} \pm(1-\mathrm{m})$ we then find in an exactly similar manner, since $3\left(\eta^{\prime} a\right)_{3 / 2}+(\eta a)_{-1 / 2}=(2,10)$ (Art. 22),

$$
3 \mu_{1}+\mu_{1}^{\prime}=(1,10) \text {. }
$$

The results $(a),\left(a^{\prime}\right)$ will be needed later.

\section{Terms of the Fourth Order.}

27. It will be sufficient, in order to prove the theorem of Art. 4, to show that these terms can be obtained accurately to $\mathrm{m}^{8}$. As all coefficients of previous orders are known to $\mathrm{m}^{9}$ except those of arguments $\pm(c-1-\mathrm{m})$ in the terms of characteristic e $e^{\prime} a$, it is only necessary to consider terms which depend on these two coefficients and terms which have small divisors equal to $P_{2}$.

The characteristic e $e^{\prime} a$ only occurs in combination with one of the characteristics e, $e^{\prime}, a$. The small divisors in the combinations e $e^{\prime} a, e^{\prime}$ and e $e^{\prime} a, a$ are equal to $P_{1}$ and the terms with characteristics $e^{\prime}, a$ are equal to $(1,10)$. We thus have, for these two combinations,

$$
(0,8)(1,10) \div(1,11)=(0,8), \mathrm{m}^{2}(0,8) \div(1,11)^{2}=(0,8) .
$$

The small divisors occurring in the combination e $e^{\prime} a$, e arise from $\tau=\mathrm{c}+P_{q}$. Referring to $(5)^{\prime}$, we see that such terms are

$$
D\left(u_{e^{\prime} e_{a}} \zeta^{-1}\right) D\left(s_{e} \zeta\right)+D\left(s_{e e^{\prime} a} \zeta^{-1}\right) D\left(u_{e} \zeta\right)
$$

which give at the worst

$$
(\mathrm{c}-1-\mathrm{m})(0,8)(0,10)=(2,10), \quad(1,9)(1,9)=(2,10),
$$

and these, divided by $(2,11)$ give $(0,8)$.

It is therefore only a question of examining terms for which $\tau=P_{2}$ or $\tau=\mathrm{c}+P_{2}$, leaving aside the parts containing the characteristic e $e^{\prime} \alpha$. In all cases these terms can be obtained to $\mathrm{m}^{7}$. Hence the contributions of terms of orders 0,4 will be at the worst

$$
(2,10)(0,7) \div(1,11)=(1,8), \quad(2,10)(0,8) \div(2,11)=(0,8),
$$

so that these combinations need not be further considered. 
28. For the terms in which $\tau=P_{2}$, the equation (11) is used. We have just seen that the terms with characteristics e $e^{\prime} a$ will not occur, and therefore the only portion which can produce results not correct to $\mathrm{m}^{8}$ will be the second term of the right-hand member. But this being of the third degree in $u-u_{0}$, $s-s_{0}, z$ can only contain terms of the first and second orders; with the exception of terms of characteristic $e^{\prime} a$, these are all known to $\mathrm{m}^{10}$. But these excepted terms can only occur with terms from two of the three characteristics $\mathrm{e}, e^{\prime}, a$, so that at the worst they occur in the combination

$$
(0,9)(0,10)(1,10) \div(2,11)=(-1,8) \text {. }
$$

The terms in $\mathrm{m}^{-1}$ disappear and all the results are known to $\mathrm{m}^{8}$.

29. The only terms in which $\tau=\mathrm{c}+P_{2}$ occur in the characteristics

$$
e^{\prime 3} a, e^{\prime} a^{3}, e^{\prime} \mathrm{k}^{2} a, \mathrm{e}^{2} \mathrm{e}^{\prime} a .
$$

The combinations of orders 1,3 , excluding e, e $e^{\prime} a$, are all at least equal to $(1,10)(0,9)=(1,10)$ so that on division by $(2,11)$ they give the results correct to $\mathrm{m}^{8}$. The only combinations of orders 2,2 which do not give results correct to $\mathrm{m}^{8}$ are $e^{\prime} a, \mathrm{k}^{2}$ and $e^{\prime} a, \mathrm{e}^{2}$, for all the others are at least equal to

$$
(0,10)(0,10)=(0,10) \text {. }
$$

For the combinations $e^{\prime} a, \mathrm{k}^{2}$ and $\mathrm{k}, \mathrm{k} e^{\prime} a$, with arguments $\pm(2 \mathrm{~g}-1-\mathrm{m})$, put $\mathrm{b}=e^{\prime} a$ in Art. 18. Then

$$
\beta, \beta^{\prime}=(0,9), \quad Q_{3}=(0,10),
$$

for $Q_{3}$ can only contain terms of the first two orders from amongst which those of characteristic $e^{\prime} a$ are excluded. All the other conditions are fulfilled and therefore the results are correct to $\mathrm{m}^{8}$.

For the same combination with arguments $1+m$, we see from the result at the end of Art. 18 that it is only necessary to consider

$$
D z_{\mathrm{k}} D z_{\mathrm{ke}^{\prime} \mathrm{a}}-D^{2}\left(z_{\mathrm{k}} z_{\mathrm{ke}^{\prime} a}\right)
$$

for the combinations of arguments $-\mathrm{g}, \mathrm{g}+1+\mathrm{m}$ and $\mathrm{g},-\mathrm{g}+1+\mathrm{m}$ since these are the only ones which will not be equal to $(1,10)$. Using for the moment, the notations of Art. 26, these terms give

$$
\left\{\mathrm{g}(\mathrm{g}+1+\mathrm{m})+(1+\mathrm{m})^{2}\right\} \mu k_{0}+\left\{\mathrm{g}(-\mathrm{g}+1+\mathrm{m})+(1+\mathrm{m})^{2}\right\} \mu^{\prime} k_{0}
$$

which is equal to

$$
\left(3 \mu+\mu^{\prime}\right) k_{0}+(1,11)(0,9)(0,10)=(1,10)(0,10)+(1,10)=(1,10),
$$


since (Art. 26) $3 \mu+\mu^{\prime}=(1,10)$. On division by $(2,11)$ the result will be ('o)rect to $\mathrm{m}^{8}$.

For the combination $\mathrm{e}^{2}, e^{\prime} a$ and arguments $\pm(2 \mathrm{c}-1-\mathrm{m})$, the only terms in $(5)^{\prime}$ which will not be true to $\mathrm{m}^{10}$ will be formed from

$$
u_{\mathrm{e}^{2}} \zeta^{-1}=\left(\epsilon^{2}\right)_{0} \zeta^{2 c}+\left({\epsilon^{\prime 2}}^{\prime 2} \zeta_{0}^{-2 c}, \quad u_{e^{\prime} a} \zeta=(\eta a)_{\frac{1}{2}} \zeta^{1+\mathrm{m}}+\left(\eta^{\prime} a\right)_{-\frac{1}{2}} \zeta^{-1-\mathrm{m}} .\right.
$$

Substituting in $(5)^{\prime}$ and neglecting a factor $(0,11)$, we find that the portion of the coefficient of $\zeta^{2 \mathrm{c}-1-\mathrm{m}}$ which arises from these terms is

$\left(\epsilon^{2}\right)_{0}(\eta a)_{1 / 2}+\left(\epsilon^{\prime 2}\right)_{0}\left(\eta^{\prime} a\right)_{-1 / 2} \equiv\left\{\left(\epsilon^{2}\right)_{0}-3\left(\epsilon^{\prime 2}\right)_{0}\right\}(\eta a)_{1 / 2}+\left(\epsilon^{\prime 2}\right)_{0}\left\{3(\eta a)_{1 / 2}+\left(\eta^{\prime} a\right)_{-3 / 3}\right\}$.

It is easy to show* that $\left(\epsilon^{2}\right)_{0}-3\left(\epsilon^{\prime 2}\right)_{0}=(1,10)$, and therefore using the result at the end of Art. 22, the expression is equal to

$$
(1,10)(0,9)+(0,10)(2,10)=(1,10) .
$$

On division by $(2,11)$ the result is true to $\mathrm{m}^{8}$.

30. For terms of orders higher than the fourth a similar treatment can be made. If we had obtained all the second order terms correctly to $\mathrm{m}^{10}$ there would have been no difficulty in obtaining all the third order terms in $u$ to $\mathrm{m}^{9}$ and those in $z$ to $\mathrm{m}^{10}$ and then no special investigations would have been required to obtain the fourth order terms in $u$ to $\mathrm{m}^{8}$ and in $z$ to $\mathrm{m}^{9}$. In fact, the transformations of Section III enable us, in the worst cases of small divisors $\left(\tau=P_{2}, \pm \tau=\mathrm{c}+P_{2}\right)$, to throw the loss of two powers of $\mathrm{m}$ on the order next but one below that under consideration. Suppose that we know the terms of order $\lambda(\lambda>3)$ in $u$ correctly to $\mathrm{m}^{q-\lambda}$ and it be required to show that the terins of order $\lambda+1$ may be obtained correctly to $\mathrm{m}^{2-\lambda-1}$. In the case of these divisors the loss of two powers of $m$ is thrown on to the terms of order $\lambda-1$ and lower; these being known to $\mathrm{m}^{q-\lambda+1}$, the results will be true to $\mathrm{m}^{q-\lambda-1}$. As the third and fourth order terms follow this rule, terms of all the following orders will do so.

The terms of order $\lambda+1$ in $z$ can be obtained to the same degree of accuracy as the terms of order $\lambda$ in $u$, that is to the order $\mathrm{m}^{q-\lambda}$.

The terms of the fourth order being all known to $\mathrm{m}^{s}$ and as we require those of the fifth order only to $\mathrm{m}^{7}$ and not to $\mathrm{m}^{8}$, the great majority of the difficulties arising from the terms of the second order in $e^{\prime} a$ will not occur after the fourth order.

No general transformation has been given for terms in which $\pm \tau=\mathrm{c}+P_{2}$. These terms must contain some power of e, $e^{\prime}, a$ in their characteristics; the

* The expression is zero in the purely elliptic formula $(\mathrm{m}=0)$ and each coefficient is known to $\mathrm{m}^{10}$. 
only cases where difficulties will occur will be when they contain e. Such difficulties can, however, be solved by the method used in Art. 25 for the terms of arguments $\pm(2-\mathrm{c}+2 \mathrm{~m})$.

31. The following table shows the results of this section in a form which is convenient for reference. In the case of the coefficients in $z \sqrt{-1}$, for $\mathrm{c}+P_{q}$ read.g $+P_{q}$.

\begin{tabular}{|c|c|c|c|}
\hline Ord. & $\tau \neq P_{q}, \pm \tau \neq \mathrm{c}+P_{q}$ & $\tau=P_{q}$ & $\pm \tau=\mathbf{c}+\boldsymbol{P}_{q}$ \\
\hline 0 & $a_{0}=1, a_{i}=(2,10)$ & none & none \\
\hline 1 & $\begin{array}{r}\varepsilon_{0}, \varepsilon_{0}^{\prime}, k_{0}=(0,10) \\
3 \varepsilon_{0}+\varepsilon_{0}^{\prime}=(2,11) \\
\text { other coeff. }=(2,10)\end{array}$ & $\eta_{0}, \eta_{0}^{\prime}=(1,10)$ & $\begin{aligned} \varepsilon_{-1}, \varepsilon_{1}^{\prime}, k_{-1} & =(1,10) \\
\varepsilon_{-1}+\varepsilon_{1}^{\prime} & =(2,10)\end{aligned}$ \\
\hline 2 & $\mid \begin{array}{r}\left(\varepsilon^{2}\right)_{0},\left(\varepsilon^{\prime z}\right)_{0},\left(\varepsilon \varepsilon^{\prime}\right)_{0}=(0,10) \\
\left(k^{\prime 2}\right)_{0},\left(k k^{\prime}\right)_{0}=(0,10) \\
\left(\varepsilon^{2}\right)_{0}-3\left(\varepsilon^{\prime 2}\right)_{0}=(0,10) \\
\left(k^{2}\right)_{0},\left(\eta^{2}\right)_{-1},\left(\eta^{\prime 2}\right)_{-1}=(2,11) \\
\text { other coeff. }=(1,10)\end{array}$ & all coeff. $=(1,10)$ & $\begin{aligned}(\eta a)_{1 / 2},\left(\eta^{\prime} a\right)_{-3 / 2} & =(0,9) \\
(\eta a)_{-1 / 2},\left(\eta^{\prime} a\right)_{1 / 2} & =(1,9) \\
3(\eta a)_{1 / 2}+\left(\eta^{\prime} a\right)_{-1 / 2} & =(2,10) \\
(\eta a)_{-1 / 2}+\left(3 \eta^{\prime} a\right)_{1 / 2} & =(2,10) \\
\text { other coeff. } & =(1,10)\end{aligned}$ \\
\hline 3 & $\begin{array}{r}\text { all coeff. }=(0,9) \\
3(k \eta a)_{1 / 2}+\left(k \eta^{\prime} a\right)_{-1 / 2}=(1,10) \\
3\left(k \eta^{\prime} a\right)_{1 / 2}+(k \eta a)_{-1 / 2}=(1,10)\end{array}$ & $\begin{array}{r}\left(\varepsilon \eta^{\prime} a\right)_{-1 / 2},\left(\varepsilon^{\prime} \eta a\right)_{1 / 2}=(0,8) \\
\text { other coeff. }=(0,9)\end{array}$ & all coeff. $=(0,9)$ \\
\hline 4 & all coeff. $=(0,8)$ & all coeff $=(0,8)$ & all coeff. $=(0,8)$ \\
\hline
\end{tabular}

32. The results of this section once proved, the practical difficulty of carrying them out is not great. Suppose that it is a question of inequalities such that $\pm \tau=\mathrm{c}+P$. Then $3 \lambda+\lambda^{\prime}$ can be and would be determined without loss of accuracy and without any increase in the number of calculations, although there may be a loss in $\lambda, \lambda^{\prime}$. In the after work we simply treat the result for $\lambda-\lambda^{\prime}$ as though it were accurate (if a numerical development is being made, an arbitrary figure is added to the last place of decimals found); it may be the function $3 \lambda+\lambda^{\prime}$ which afterwards really arises. If it be so, the future results will be correct to the orders stated above.

When this method cannot be followed, it is usually best to find the coefficients as accurately as possible by the ordinary methods and then treat the special coefficients in which there has been a loss of accuracy by the special methods. The case of $\epsilon_{-1}, \epsilon_{1}^{\prime}$ (Art. 21) is an example of this.

Haverford College, Pa. 